\title{
Water-extractable organic matter from plant lit- ter and soil of rough fescue grassland
}

\author{
JOHAN F. DORMAAR AND WALTER D. WILLMS \\ Authors are soil scientist and range ecologist, respectively, Agriculture Canada Research Station, Lethbridge, Alta. \\ $T 1 J 4 B I$.
}

\begin{abstract}
Little is known about the chemical composition of throughfall, or the water that falls through, and drips from, the grass canopy of Rough Fescue Grassland during the grazing season. Waterextractable $\mathbf{C}, \mathbf{N}$, organic acids, and monosaccharides from litter and from soil in the upper $2 \mathrm{~cm}$ of the Ah horizon collected at monthly intervals in 1988 were assessed at Stavely, Alberta. Rough fescue (Festuca campestris Rydb.) grasslands were stocked at either light (1.2 AUM/ha) or very heavy (4.8 AUM/ha) fixed rates for 39 years or were ungrazed in exclosures located within each field for an equal period of time. At the high grazing intensity, the soil and litter $\mathbf{N}$ was less water-extractable. The $\mathrm{C} / \mathrm{N}$ ratios of the water-extractable organic matter from litter and soil averaged 11.2 and 2.3, respectively. Soil monosaccharides were essentially not water-extractable. The quality of the litter as reflected by the water-extractable constituents often differed over the season between fields. Observations at regular time intervals are essential. The effect of the quality of leachates of litter on soil was not predictable. The 3 major long-chain fatty acids identified, palmitic, stearic, and arachidic acids, from soil in grasslands that are in good condition because of the low grazing pressure, could well contribute to the resistance of those grasslands to the encroachment of invading species.
\end{abstract}

Key Words: soil quality, soil chemical properties, monosaccharides, organic acids, stocking rate, chernozemic.

Litter is that part of the forage resource not produced in the current year. Dead plant material, found in the plant canopy (standing litter) or on the soil (fallen litter), has a large influence on the productivity, biomass accumulation, and species composition of plants and animals (Knapp and Seastedt 1986). The microenvironment of grasslands is significantly modified by plant litter (Willms et al. 1986, Willms 1988), which increases water infiltration, reduces radiation density, and insulates the soil surface, thereby keeping soil temperatures cooler in spring and summer while reducing evaporation.

Grazing affects the quantity and quality of litter on grasslands. Its long-term effect, the alteration of species composition, is achieved by shifting the competitive advantage to species that are

\footnotetext{
Contribution No. 3879049. The technical assistance of R.R. James is greatly appreciated.

Manuscript accepted 25 May 1991.
}

smaller, more shallow rooted, and less productive, but more resistant to grazing and more tolerant of drier soil conditions resulting from litter removal. Therefore, while litter accumulation is affected by grazing over the short term, litter production and, presumably, quality are affected over the long term by the new species composition. Litter quantity on the rough fescue grassland was reduced from $12,403 \mathrm{~kg} /$ ha on ungrazed range to $247 \mathrm{~kg} /$ ha on heavily grazed range (Peake and Johnston 1965) while the average percentage basal area of the dominant rough fescue (Festuca campestris Rydb.) decreased from 7.5 to 0.6 for the same grazing treatments, respectively (Johnston et al. 1971).

Plant canopies in the natural environment can alter the chemical composition of precipitation falling through them. For example, litter affects the chemistry of nitrogen in rainwater passing through it (Knapp and Seastedt 1986, Gilliam 1987). The chemical properties of soil under litter will be affected both by the quality and quantity of litter aboveground and by the chemical composition of the rainwater falling through the litter. During the decomposition of litter by abiotic and biotic agents, many hydroxyl-and carboxylcontaining compounds are released and leached into the soil by precipitation. Their presence in the soil has both detrimental and beneficial ecological consequences (Whittaker 1970, Rice 1984). Components of extracts from grassland litter exhibit phytotoxic effects on the germination of various range grasses (Johnston 1961, Bokhari 1978) and affect competition among species (Rice 1984).

Little is known about the chemical composition of the water that falls through, and drips from, the grass canopy (throughfall) of rough fescue grassland during the grazing season, particularly as it is affected by different grazing pressures. Also there is little information available on possible differences between water extracts of litter and of soil immediately below the surface. This study was conducted to examine the potential contribution of litter to the chemical composition of water extracts during the grazing season, and to assess the relationship of water-extractable constituents from litter and the top $2 \mathrm{~cm}$ of soil under various grazing pressures.

\section{Materials and Methods}

\section{Sampling Sites}

The study site was at the Agriculture Canada Research Substation, Stavely, Alberta, situated in the Porcupine Hills, an area managed primarily for grazing by cattle. The vegetation is typical of the Fescue Grassland Association (Coupland and Brayshaw 
1953). The soils are members of the Orthic Black Subgroup of the Chernozemic Order (Udic Haploboroll) developed on till overlying sandstone, and have a clay-loam to loam texture. The climate is dry subhumid and annual precipitation averages about $500 \mathrm{~mm}$. Details of the overall grazing trial ongoing since 1949 have been given by Johnston et al. (1971) and Willms et al. (1985).

The study was conducted in 1988 in 2 fields stocked from midMay to mid-November, at either light (field L) or very heavy (field VH) rates, and in their ungrazed exclosures (fields E) to provide 3 grazing treatments. Field $\mathrm{L}$ has been stocked at 1.2 animal unit months (AUM)/ha since 1949; field VH was stocked at 4.8 $\mathrm{AUM} /$ ha from 1949 to 1958 . Since 1959, the stocking rate on field VH averaged 3.2 AUM/ha and varied, depending on annual precipitation, from 2.4 to $4.8 \mathrm{AUM} /$ ha. Cattle were removed from field VH when utilization was about $80 \%$ of annual production. Average utilization in field $\mathrm{L}$ was about $25 \%$. The exclosures, 0.5 ha in area, were constructed in 1948 and have been protected from livestock grazing since then. Percent composition (basal area) of fields $\mathrm{L}$ and $\mathrm{VH}$ and of the exclosures was presented in a previous study (Willms et al. 1985).

\section{Sampling}

Grassland litter, consisting of all dead herbage, standing as well as the accumulated mulch, and soil from the upper $2 \mathrm{~cm}$ of the Ah horizon immediately below the litter were sampled each month from May to November, 1988, in 3 randomly located sub-plots in each field and within each exclosure. To reduce the number of samples requiring analysis, 3 subsamples from 1 exclosure were randomly paired with 3 subsamples from the second exclosure and pooled to give 3 common exclosure subsamples.

The soil samples were dried and ground to pass a $0.5-\mathrm{mm}$ sieve. At the time of sieving, roots and other debris were removed from the soil and discarded. The litter was dried at $60^{\circ} \mathrm{C}$ for 24 hours and ground in a Wiley mill to pass through a 100-mesh sieve. Ash content of the ground litter was determined on a portion of each sample by ignition at $700^{\circ} \mathrm{C}$ for 4 hours.

\section{Analyses \\ Chemical}

Total $\mathrm{C}$ and $\mathrm{N}$ were determined to enable the calculation of water-extractable $\mathrm{C}$ and $\mathrm{N}$ as a percentage of litter and soil $\mathrm{C}$ and $\mathrm{N}$. Total organic $\mathrm{C}$ was determined by dry combustion at $900^{\circ} \mathrm{C}$ for $15 \mathrm{~min}$; the evolved $\mathrm{CO}_{2}$ was collected and weighed. Total $\mathrm{N}$ was determined by the Kjeldahl procedure as outlined by the Association of Official Agricultural Chemists (1950).

To simulate maximum leachates of organic acids from litter and soil, 1 gram of material and $15 \mathrm{ml}$ of distilled water were placed in 50 -ml polypropylene centrifuge tubes. After shaking in a circulating shaker for $\mathbf{4 8}$ hours, the mixture was centrifuged and filtered. The remaining litter or soil was washed with an additional $15 \mathrm{ml}$ of water, centrifuged, and filtered. The 2 supernatant solutions were combined and the $\mathrm{pH}$ recorded. The combined filtrates were extracted with $60 \mathrm{ml}$ dichloromethane $\left(\mathrm{CH}_{2} \mathrm{Cl}_{2}\right)$ in a separatory funnel. The lower fraction, consisting of $\mathrm{CH}_{2} \mathrm{Cl}_{2}$, was drawn off and dried over anhydrous $\mathrm{Na}_{2} \mathrm{SO}_{4}$; the upper water layer was saved for a second $\mathrm{CH}_{2} \mathrm{Cl}_{2}$ extraction at $\mathrm{pH} 2.5$.

The water-extractable organic acids were determined by a modification of a procedure outlined by Dormaar and Willms (1990). This first $\mathrm{CH}_{2} \mathrm{Cl}_{2}$-extracted fraction was reduced to about $3 \mathrm{ml}$, transferred to a small vial and further reduced to $250 \mu 1$. Fifty microliters of a vanillic acid solution at a concentration of 0.2 $\mu \mathrm{g} / \mu \mathrm{l}$ was added to serve as internal standard. The mixture was then evaporated to dryness at $40^{\circ} \mathrm{C}$ with a stream of nitrogen. To obtain silylation, $150 \mu 1$ pyridine and $50 \mu 1 \mathrm{~N}, 0$-bis(trimethylsilyl) tri-fluoroacetamide (BSTFA) were added and mixed. After standing for $10 \mathrm{~min}$ in the closed vial, the sample was ready for gas chromatographic analysis.

The $\mathrm{pH}$ of the water phase, after the first $\mathrm{CH}_{2} \mathrm{Cl}_{2}$ extraction was drawn off, was readjusted to $\mathrm{pH} 2.5$ with $\mathrm{HCl}$ and extracted once again with $\mathrm{CH}_{2} \mathrm{Cl}_{2}$ after which this second $\mathrm{CH}_{2} \mathrm{Cl}_{2}$ extract was processed like the first $\mathrm{CH}_{2} \mathrm{Cl}_{2}$ extract in preparation for gas chromatographic analysis.

The quantitative analyses were carried out with a Hewlett Packard GC 5840A using a 30-m long fused silica capillary column wall-coated with cross-linked methylsilicone at a film thickness of $0.5 \mu$. Total water-extractable organic acids were calculated up to retention time 27.05 minutes. The cumulative ( $\mathrm{pH}$ as extracted and $\mathrm{pH}$ at 2.5) quantitative output of the 3 major peaks, 2 of which were easily identified, also were calculated.

For water-extractable carbohydrates, the initial water extraction from the litter and the soil was similar to that described under water-extractable organic acids. After the $\mathrm{pH}$ values of the extracts were recorded, the $\mathrm{pH}$ was adjusted to 7.5 with $0.1 \mathrm{~N} \mathrm{HCl}$. Sugars in the solution were reduced, acetylated, and analyzed as outlined by Dormaar (1984). Fucose acetate was used as internal standard because significant amounts of sugar alcohol inositol were present in the water extracts of the litter. Deoxyhexoses (rhamnose), pentoses (ribose + arabinose + xylose), hexoses (mannose + galactose + glucose), and inositol were expressed as $\mu \mathrm{g} / \mathrm{g}$, but their totals were expressed as $\mathrm{mg} / \mathrm{g}$.

\section{Statistical}

All analytical results were expressed on the basis of oven-dried, ash-free weight of litter and oven-dried weight of soil.

Although replication and application of current statistical analyses to newly established, replicated field plot experiments is common and undeniably desirable and useful, valid information and data can still be gained from early established, unreplicated field experiments including long-term grazing trials, by virtue of their antiquity (Ridley and Hedlin 1968, Dormaar and Pittman 1980).

The concentration of each organic constituent was compared across grazing treatments using analysis of variance with the experimental error derived from the sampling error. Single degree of freedom contrasts (Steel and Torrie 1980) were used to test for differences between paired means. Individual analyses were made by sampling month and medium type (soil or litter).

The relationship between specific organic constituents in litter and soil was examined within each grazing treatment using regression analysis. Simple coefficients were calculated for concentrations from paired litter $(x)$ and soil (y) samples over subsamples (3) and sampling months (6) for a total of 18 paired values after accounting for the effect of time (month). The effect of time (month) on the concentration of each constituent was examined with 1st or 2nd degree polynomial regressions.

\section{Results}

Tables 1,2, and 3 present the original data and the results of the contrast tests for differences between selected means and 1 st and 2nd degree polynomial regressions over month. Generally, all data show seasonal trends and many of the contrasts are either significant $(P<0.05)$ or highly significant $(P<0.01)$. Most regressions were 2 nd degree polynomials, with mid-seasonal maxima.

The $\mathrm{pH}$ of the water extracts of the litter showed more significant contrasts than that of the soil (Table 1 ). The $\mathrm{C}$ content of the soil extracts showed the same trends as in a previous study examining the whole Ah horizon (Dormaar et al. 1984) but with larger values because this study examined only the upper $2 \mathrm{~cm}$ of the $A h$ horizon. As grazing intensity increased, the concentration of total $\mathrm{N}$ generally increased and the water-extractable $\mathrm{N}$ as a percent of total $\mathbf{N}$ decreased. 
Table 1. Carbon and nitrogen contents (\%) of litter and soil and of their water extracts sampled in 1988 from 2 fields stocked at either light or heavy rates and from their exclosures $(n=3)$.

\begin{tabular}{|c|c|c|c|c|c|c|c|c|c|c|c|c|}
\hline & \multicolumn{6}{|c|}{ Litter } & \multicolumn{6}{|c|}{ Soil } \\
\hline & \multirow{2}{*}{$\begin{array}{l}\text { Exclosures } \\
\text { (E) }\end{array}$} & \multicolumn{2}{|c|}{$\begin{array}{c}\text { Stocking } \\
\text { rate }^{1}\end{array}$} & \multicolumn{3}{|c|}{ Contrasts } & \multirow{2}{*}{$\begin{array}{l}\text { Exclosures } \\
\text { (E) }\end{array}$} & \multicolumn{2}{|c|}{$\begin{array}{c}\text { Stocking } \\
\text { rate }\end{array}$} & \multicolumn{3}{|c|}{ Contrasts } \\
\hline & & $\mathbf{L}$ & VH & E vs. L & E vs. VH & L vs. VH & & $\mathbf{L}$ & VH & E vs. $\mathbf{L}$ & E vs. VH & L vs. VH \\
\hline \multicolumn{13}{|l|}{ Total C } \\
\hline May & 45.9 & 46.9 & 45.3 & 0.006 & 0.050 & $<0.001$ & 16.6 & 15.6 & 14.2 & 0.006 & $<0.001$ & 0.001 \\
\hline June & 45.6 & 46.1 & 45.1 & 0.054 & 0.066 & 0.004 & 16.5 & 15.5 & 13.1 & 0.012 & $<0.001$ & $<0.001$ \\
\hline July & 45.5 & 46.1 & 45.1 & 0.031 & 0.095 & 0.003 & 15.0 & 13.7 & 11.4 & 0.006 & $<0.001$ & $<0.001$ \\
\hline August & 44.6 & 46.4 & 44.5 & $<0.001$ & 0.725 & $<0.001$ & 14.9 & 12.3 & 10.6 & $<0.001$ & $<0.001$ & $<0.001$ \\
\hline September & 44.3 & 46.8 & 44.1 & $<0.001$ & 0.261 & $<0.001$ & 15.2 & 12.7 & 11.9 & $<0.001$ & $<0.001$ & 0.020 \\
\hline $\begin{array}{l}\text { October } \\
P^{2}\end{array}$ & 45.4 & $\begin{array}{l}46.8 \\
* *\end{array}$ & $\begin{array}{l}45.0 \\
* 3\end{array}$ & 0.003 & 0.173 & 0.001 & 15.3 & 13.9 & 12.5 & 0.001 & $<0.001$ & 0.001 \\
\hline \multicolumn{13}{|c|}{$\begin{array}{l}\text { Water-extractable } \\
\mathrm{C} \text { as \% of total C }\end{array}$} \\
\hline May & 3.1 & 4.2 & 3.4 & 0.001 & 0.144 & 0.003 & 0.84 & 1.13 & 1.16 & $<0.001$ & $<0.001$ & 0.057 \\
\hline June & 3.5 & 4.6 & 3.9 & $<0.001$ & 0.029 & $<0.001$ & 0.88 & 1.14 & 1.18 & $<0.001$ & $<0.001$ & 0.201 \\
\hline July & 4.4 & 5.2 & 4.8 & 0.003 & 0.063 & 0.048 & 0.92 & 1.14 & 1.19 & $<0.001$ & $<0.001$ & 0.018 \\
\hline August & 4.9 & 5.8 & 5.6 & 0.004 & 0.011 & 0.343 & 0.97 & 1.42 & 1.58 & $<0.001$ & $<0.001$ & $<0.001$ \\
\hline September & 3.9 & 4.7 & 4.1 & 0.002 & 0.382 & 0.005 & 0.68 & 1.16 & 1.21 & $<0.001$ & $<0.001$ & 0.076 \\
\hline October & 3.0 & 3.6 & 3.2 & 0.011 & 0.353 & 0.040 & 0.68 & 1.05 & 1.15 & $<0.001$ & $<0.001$ & 0.016 \\
\hline$P$ & ** & $* *$ & $* *$ & & & & $* *$ & $\neq *$ & $*$ & & & \\
\hline \multicolumn{13}{|l|}{ Total N } \\
\hline May & 1.28 & 1.06 & 1.92 & 0.002 & $<0.001$ & $<0.001$ & 1.44 & 1.43 & 1.32 & 0.854 & 0.014 & 0.017 \\
\hline June & 1.15 & 1.34 & 1.96 & $<0.001$ & $<0.001$ & $<0.001$ & 1.35 & 1.32 & 1.31 & 0.260 & 0.138 & 0.657 \\
\hline July & 0.90 & 1.89 & 2.04 & $<0.001$ & $<0.001$ & 0.001 & 1.31 & 1.31 & 1.47 & 1.000 & 0.009 & 0.009 \\
\hline August & 0.89 & 1.96 & 2.13 & $<0.001$ & $<0.001$ & 0.001 & 1.07 & 1.25 & 1.43 & 0.001 & $<0.001$ & 0.001 \\
\hline September & 0.82 & 1.04 & 2.23 & 0.001 & $<0.001$ & $<0.001$ & 1.06 & 1.27 & 1.31 & $<0.001$ & $<0.001$ & 0.201 \\
\hline October & 0.70 & 0.89 & 2.12 & 0.001 & $<0.001$ & $<0.001$ & 1.25 & 1.27 & 1.31 & 0.372 & 0.048 & 0.180 \\
\hline$P$ & $*$ & $* *$ & & & & & $* *$ & $* *$ & $* *$ & & & \\
\hline \multicolumn{13}{|c|}{$\begin{array}{l}\text { Water-extractable } \\
\mathrm{N} \text { as \% of total } \mathbf{N}\end{array}$} \\
\hline May & 11.6 & 10.1 & 7.4 & $<0.001$ & $<0.001$ & $<0.001$ & 1.17 & 0.74 & 0.52 & $<0.001$ & $<0.001$ & $<0.001$ \\
\hline June & 16.4 & 12.9 & 8.4 & $<0.001$ & $<0.001$ & $<0.001$ & 0.96 & 0.74 & 0.44 & $<0.001$ & $<0.001$ & $<0.001$ \\
\hline July & 18.8 & 15.7 & 8.9 & $<0.001$ & $<0.001$ & $<0.001$ & 0.78 & 0.63 & 0.43 & 0.004 & $<0.001$ & 0.001 \\
\hline August & 22.0 & 20.6 & 9.8 & 0.008 & $<0.001$ & $<0.001$ & 0.51 & 0.41 & 0.16 & 0.010 & $<0.001$ & $<0.001$ \\
\hline September & 18.2 & 12.7 & 11.2 & $<0.001$ & $<0.001$ & $<0.001$ & 0.56 & 0.40 & 0.63 & 0.003 & 0.070 & $<0.001$ \\
\hline October & 17.8 & 12.4 & 9.6 & $<0.001$ & $<0.001$ & $<0.001$ & 0.63 & 0.80 & 0.65 & 0.002 & 0.642 & 0.004 \\
\hline$P$ & $* *$ & $* *$ & $* *$ & & & & $* *$ & $\omega *$ & $* *$ & & & \\
\hline \multicolumn{13}{|c|}{$\begin{array}{l}\mathrm{pH} \text { of the } \\
\text { water extract }\end{array}$} \\
\hline May & 5.8 & 5.9 & 6.2 & 0.604 & 0.001 & 0.002 & 7.0 & 7.0 & 7.1 & 0.725 & 0.310 & 0.488 \\
\hline June & 6.0 & 5.8 & 6.1 & 0.001 & 0.506 & 0.001 & 7.3 & 7.1 & 7.1 & 0.005 & 0.009 & 0.604 \\
\hline July & 5.9 & 5.5 & 5.8 & 0.001 & 0.152 & 0.005 & 7.5 & 7.5 & 7.6 & 0.604 & 0.315 & 0.604 \\
\hline August & 5.9 & 5.4 & 5.8 & $<0.001$ & 0.071 & $<0.001$ & 7.4 & 7.7 & 7.5 & 0.035 & 0.522 & 0.088 \\
\hline September & 5.6 & 5.7 & 5.8 & 0.315 & 0.034 & 0.152 & 7.3 & 7.3 & 7.6 & 1.000 & 0.010 & 0.010 \\
\hline October & 5.6 & 5.9 & 6.1 & 0.001 & $<0.001$ & 0.005 & 7.2 & 7.3 & 7.3 & 0.315 & 0.152 & 0.604 \\
\hline$P$ & $* *$ & $* *$ & $* *$ & & & & $* *$ & $* *$ & $* *$ & & & \\
\hline
\end{tabular}

iLight (L) - stocked at 1.2 AUM/ha; Very Heavy (VY) - stocked at 4.8 AUM/ha.

2Level of significance $(*=P<0.05, * *=P<0.01)$ of 2 nd degree polynomial regressions of the constituent concentration over month.

3Only 1 st degree polynomial regression.

The $\mathrm{C} / \mathrm{N}$ ratios of litter when averaged over the season were 49.2, 37.4, and 21.8 for fields $\mathrm{E}, \mathrm{L}$, and $\mathrm{VH}$, respectively. These values were, respectively, $11.8,10.6$, and 9.1 for the soil; $10.6,12.9$, and 10.1 for water-extractable organic matter from the litter; and 1.4, 2.2, and 3.2 for water-extractable organic matter from the soil.

There were more nonsignificant contrasts for organic acids at the $\mathrm{pH}$ as extracted from the soil than for the subsequent $\mathrm{pH}$ modified extract (Table 2). Most of the water-extractable organic acids were obtained at the $\mathrm{pH}$ of the original extract; additional organic acids for the soil extracts were obtained at $\mathrm{pH} 2.5$.

The quality of the litter in terms of water-extractable quantities of monosaccharides differed significantly among grazing treatments (Table 3). Generally, pentoses $>$ hexoses $>$ deoxyhexoses, which meant low galactose + mannose / xylose + arabinose ratios. There was also, generally, more mannose and galactose from the litter of field $\mathrm{L}$ than from field $\mathrm{VH}$, while the reverse was true for arabinose and xylose. Conversely, the soil essentially did not release any measurable water-extractable monosaccharides; hence, the data are not presented in Table 3.

Effect of month was highly significant for each constituent shown in Tables 1 and 2 and particularly for those shown in Table 3. Only 6 regressions of constituent in soil vs. litter were significant after removing the effect of time (Table 4). The coefficient describing the relationship between constituent in soil vs. litter was often negative, but significant $(P<0.05)$ in only 2 instances, (waterextractable $\mathrm{N}$ as \% of total $\mathrm{N}$ for lightly grazed; organic acids at $\mathrm{pH}$ 2.5 for exclosure).

\section{Discussion}

The effect of grazing on basal cover under various grazing pressures has been well-documented (Peake and Johnston 1965, 
Table 2. Organic acids $(\mu \mathrm{g} / \mathrm{g})$ in water extracts of litter and soil sampled in 1988 from 2 fields stocked at either light or very heavy rates and from their exclosures $(\mathbf{n}=3)$.

\begin{tabular}{|c|c|c|c|c|c|c|c|c|c|c|c|c|}
\hline & \multicolumn{6}{|c|}{ Litter } & \multicolumn{6}{|c|}{ Soil } \\
\hline & \multicolumn{2}{|c|}{ Exclosures } & $\begin{array}{c}\text { Stocking } \\
\text { rate }^{1}\end{array}$ & \multicolumn{3}{|c|}{ Contrasts } & Exclosures & \multicolumn{2}{|c|}{$\begin{array}{c}\text { Stocking } \\
\text { rate }\end{array}$} & \multicolumn{3}{|c|}{ Contrasts } \\
\hline & (E) & $\mathbf{L}$ & VH & E vs. L & E vs. VH & L vs. VH & (E) & $\mathbf{L}$ & VH & E vs. $L$ & E vs. VH & L vs. VH \\
\hline \multicolumn{13}{|c|}{$\begin{array}{l}\text { Organic acids } \\
\text { at } \mathrm{pH} \text { as extracted }\end{array}$} \\
\hline May & 210 & 101 & 125 & $<0.001$ & $<0.001$ & 0.002 & 62 & 60 & 66 & 0.387 & 0.090 & 0.025 \\
\hline June & 264 & 207 & 193 & $<0.001$ & $<0.001$ & 0.004 & 120 & 118 & 78 & 0.627 & $<0.001$ & $<0.001$ \\
\hline July & 425 & 207 & 284 & $<0.001$ & $<0.001$ & $<0.001$ & 82 & 65 & 69 & 0.001 & 0.003 & 0.190 \\
\hline August & 361 & 311 & 221 & $<0.001$ & $<0.001$ & $<0.001$ & 53 & 50 & 52 & 0.065 & 0.482 & 0.184 \\
\hline September & 299 & 219 & 223 & $<0.001$ & $<0.001$ & 0.194 & 46 & 33 & 38 & $<0.001$ & 0.003 & 0.026 \\
\hline $\begin{array}{l}\text { October } \\
P^{2}\end{array}$ & $\begin{array}{l}253 \\
* * *\end{array}$ & $\begin{array}{l}147 \\
* *\end{array}$ & 202 & $<0.001$ & $<0.001$ & $<0.001$ & $2^{27}$ & $\underset{+43}{26}$ & 27 & 0.715 & 0.715 & 0.473 \\
\hline \multicolumn{13}{|c|}{$\begin{array}{l}\text { Organic acids } \\
\text { at } \mathrm{pH} 2.5^{4}\end{array}$} \\
\hline May & 51 & 32 & 49 & $<0.001$ & $<0.001$ & 0.012 & 21 & 12 & 9 & $<0.001$ & $<0.001$ & 0.076 \\
\hline June & 89 & 76 & 81 & 0.001 & 0.011 & 0.071 & 114 & 25 & 18 & $<0.001$ & $<0.001$ & 0.043 \\
\hline July & 93 & 79 & 112 & 0.001 & $<0.001$ & $<0.001$ & 57 & 23 & 23 & $<0.001$ & $<0.001$ & 0.680 \\
\hline August & 91 & 81 & 110 & 0.010 & 0.001 & $<0.001$ & 31 & 35 & 27 & 0.069 & 0.040 & 0.003 \\
\hline September & 61 & 58 & 104 & 0.133 & $<0.001$ & $<0.001$ & 24 & 76 & 37 & $<0.001$ & 0.002 & $<0.001$ \\
\hline October & 45 & 51 & 94 & 0.062 & $<0.001$ & $<0.001$ & 32 & 20 & 18 & $<0.001$ & $<0.001$ & 0.108 \\
\hline$P$ & $* *$ & $* *$ & $* *$ & & & & NS & $* 3$ & $* *$ & & & \\
\hline \multicolumn{13}{|c|}{$\begin{array}{l}\text { Palmitic acid } \\
\text { (at pH as extracted } \\
+ \text { at } \mathrm{pH} 2.5)^{5}\end{array}$} \\
\hline May & 10 & 5 & 5 & $<0.001$ & $<0.001$ & 0.012 & 15 & 15 & 12 & 0.828 & 0.087 & 0.063 \\
\hline June & 14 & 6 & 7 & $<0.001$ & 0.001 & 0.453 & 33 & 21 & 14 & $<0.001$ & $<0.001$ & 0.006 \\
\hline July & 16 & 15 & 21 & 0.258 & 0.018 & 0.004 & 19 & 19 & 15 & 1.000 & 0.020 & 0.020 \\
\hline August & 21 & 12 & 17 & $<0.001$ & 0.009 & 0.003 & 15 & 18 & 17 & 0.128 & 0.168 & 0.851 \\
\hline September & 18 & 9 & 12 & $<0.001$ & $<0.001$ & 0.010 & 11 & 17 & 10 & 0.012 & 0.439 & 0.005 \\
\hline October & 12 & 4 & 5 & $<0.001$ & $<0.001$ & 0.315 & 7 & 5 & 9 & 0.034 & 0.017 & 0.001 \\
\hline$P$ & $* *$ & $* *$ & $* *$ & & & & $*$ & $* *$ & $* *$ & & & \\
\hline \multicolumn{13}{|c|}{$\begin{array}{l}\text { Stearic acid } \\
\text { (at pH as extracted } \\
+ \text { at } \mathrm{pH} 2.5 \text { ) }\end{array}$} \\
\hline May & 16 & 8 & 9 & $<0.001$ & $<0.001$ & 0.488 & 19 & 19 & 7 & 0.849 & $<0.001$ & $<0.001$ \\
\hline June & 18 & 14 & 10 & 0.012 & $<0.001$ & 0.005 & 36 & 21 & 18 & $<0.001$ & $<0.001$ & 0.275 \\
\hline July & 24 & 16 & 18 & 0.004 & 0.002 & 0.160 & 25 & 21 & 17 & 0.090 & 0.006 & 0.071 \\
\hline August & 24 & 24 & 17 & 0.665 & 0.004 & 0.002 & 20 & 31 & 20 & $<0.001$ & 1.000 & $<0.001$ \\
\hline September & 19 & 16 & 15 & 0.057 & 0.024 & 0.546 & 12 & 20 & 7 & $<0.001$ & 0.003 & $<0.001$ \\
\hline October & 12 & 12 & 13 & 1.000 & 0.134 & 0.134 & 4 & 3 & 5 & 0.152 & 0.071 & 0.009 \\
\hline$P$ & $* *$ & $* *$ & $* *$ & & & & $* *$ & $* *$ & $* *$ & & & \\
\hline \multicolumn{13}{|c|}{$\begin{array}{l}\text { Arachidic acid } \\
\text { (at pH as extracted } \\
+ \text { at } \mathrm{pH} 2.5)^{5}\end{array}$} \\
\hline May & 28 & 19 & 19 & $<0.001$ & $<0.002$ & 0.550 & 5 & 6 & 7 & 0.537 & 0.153 & 0.364 \\
\hline June & 48 & 35 & 25 & 0.002 & $<0.001$ & 0.006 & 14 & 6 & 5 & $<0.001$ & $<0.001$ & 0.697 \\
\hline July & 50 & 58 & 35 & 0.013 & 0.001 & $<0.001$ & 13 & 7 & 10 & 0.001 & 0.019 & 0.019 \\
\hline August & 94 & 47 & 36 & $<0.001$ & $<0.001$ & 0.194 & 7 & 19 & 16 & $<0.001$ & 0.003 & 0.091 \\
\hline September & 90 & 46 & 34 & $<0.001$ & $<0.001$ & 0.002 & 9 & 16 & 9 & 0.001 & 0.776 & 0.001 \\
\hline October & 25 & 21 & 22 & 0.003 & 0.010 & 0.267 & 23 & 8 & 20 & $<0.002$ & 0.215 & 0.001 \\
\hline$P$ & $* *$ & $* *$ & $* *$ & & & & $\star 3$ & $\star$ & $* * 3$ & & & \\
\hline
\end{tabular}

lLight (L) - stocked at 1.2 AUM/ha; Very Heavy (VH) - stocked at $4.8 \mathrm{AUM} /$ ha.

2Level of significance $(*=P<0.05, * *=P<0.01, \mathrm{NS}=P>0.05)$ of 2 nd degree polynomial regressions of the constituent concentration over month. ${ }^{3}$ lst degree polynomial regression.

ipH of water extract readjusted to 2.5 after first $\mathrm{CH}_{2} \mathrm{Cl}_{2}$ separation.

s'sum of the $2 \mathrm{CH}_{2} \mathrm{Cl}_{2}$ separations. 
Table 3. Monosaccharides and inositol in water extracts of litter sampled in 1988 from 2 fields stocked at either light or very heavy rates and from their exclosures $(n=3)$.

\begin{tabular}{|c|c|c|c|c|c|c|}
\hline & \multirow{2}{*}{$\begin{array}{l}\text { Exclosures } \\
\text { (E) }\end{array}$} & \multicolumn{2}{|c|}{ Stocking rate ${ }^{1}$} & \multicolumn{3}{|c|}{ Contrasts } \\
\hline & & L & VH & E vs. L & E vs. VH & L vs. VH \\
\hline \multicolumn{7}{|c|}{ Total (mg/g) } \\
\hline May & 0.7 & 2.5 & 3.1 & $<0.001$ & $<0.001$ & 0.001 \\
\hline June & 1.5 & 2.9 & 2.8 & $<0.001$ & $<0.001$ & 0.283 \\
\hline July & 9.3 & 12.6 & 7.3 & $<0.001$ & $<0.001$ & $<0.001$ \\
\hline August & 6.5 & 3.0 & 6.5 & $<0.001$ & 1.000 & $<0.001$ \\
\hline September & 2.1 & 2.5 & 5.6 & 0.008 & $<0.001$ & $<0.001$ \\
\hline October & 0.8 & 0.5 & 2.5 & 0.005 & $<0.001$ & $<0.001$ \\
\hline$P^{2}$ & $* *$ & *3 & $* *$ & & & \\
\hline \multicolumn{7}{|c|}{ Deoxyhexoses $(\mu \mathrm{g} / \mathrm{g})$} \\
\hline May & 26 & 49 & 122 & $<0.001$ & $<0.001$ & $<0.001$ \\
\hline June & 73 & 29 & 138 & $<0.001$ & $<0.001$ & $<0.001$ \\
\hline July & 467 & 378 & 147 & $<0.001$ & $<0.001$ & $<0.001$ \\
\hline August & 323 & 119 & 194 & $<0.001$ & $<0.001$ & $<0.001$ \\
\hline September & 147 & 253 & 223 & $<0.001$ & $<0.001$ & 0.002 \\
\hline October & 38 & 10 & 74 & $<0.001$ & $<0.001$ & $<0.001$ \\
\hline$P$ & ** & NS & $* *$ & & & \\
\hline \multicolumn{7}{|c|}{ Pentoses $(\mu \mathrm{g} / \mathrm{g})$} \\
\hline May & 237 & 1480 & 2331 & $<0.001$ & $<0.001$ & $<0.001$ \\
\hline June & 763 & 1848 & 1881 & $<0.001$ & $<0.001$ & 0.720 \\
\hline July & 5227 & 7938 & 4620 & $<0.001$ & $<0.001$ & $<0.001$ \\
\hline August & 4591 & 2136 & 4527 & $<0.001$ & 0.737 & $<0.001$ \\
\hline September & 1281 & 1875 & 4286 & $<0.001$ & $<0.001$ & $<0.001$ \\
\hline October & 399 & 355 & 2072 & 0.348 & $<0.001$ & $<0.001$ \\
\hline$P$ & ** & NS & ** & & & \\
\hline \multicolumn{7}{|c|}{ Hexoses $(\mu \mathrm{g} / \mathrm{g})$} \\
\hline May & 267 & 863 & 337 & $<0.001$ & 0.046 & $<0.001$ \\
\hline June & 543 & 997 & 581 & $<0.001$ & 0.393 & $<0.001$ \\
\hline July & 3080 & 4032 & 2053 & $<0.001$ & $<0.001$ & $<0.001$ \\
\hline August & 1229 & 653 & 1487 & $<0.001$ & 0.004 & $<0.001$ \\
\hline September & 567 & 304 & 891 & $<0.001$ & $<0.001$ & $<0.001$ \\
\hline October & 322 & 125 & 222 & $<0.001$ & 0.001 & 0.001 \\
\hline$P$ & ** & NS & ** & & & \\
\hline \multicolumn{7}{|c|}{ Inositol $(\mu \mathrm{g} / \mathrm{g})$} \\
\hline May & 27 & 74 & 276 & $<0.001$ & $<0.001$ & $<0.001$ \\
\hline June & 88 & 59 & 166 & 0.008 & $<0.001$ & $<0.001$ \\
\hline July & 560 & 252 & 513 & $<0.001$ & 0.002 & $<0.001$ \\
\hline August & 323 & 59 & 259 & $<0.001$ & $<0.001$ & $<0.001$ \\
\hline September & 105 & 101 & 167 & 0.386 & $<0.001$ & $<0.001$ \\
\hline October & 8 & 10 & 99 & $<0.001$ & 0.001 & 0.001 \\
\hline $\boldsymbol{P}$ & $*$ & NS & ** & & & \\
\hline \multicolumn{7}{|c|}{$($ Galactose + mannose $) /($ xylose + arabinose $)(\mathrm{mg} / \mathrm{g})$} \\
\hline May & 0.72 & 0.55 & 0.12 & $<0.001$ & $<0.001$ & $<0.001$ \\
\hline June & 0.67 & 0.50 & 0.29 & $<0.001$ & $<0.001$ & $<0.001$ \\
\hline July & 0.56 & 0.47 & 0.41 & 0.006 & 0.001 & 0.048 \\
\hline August & 0.24 & 0.28 & 0.30 & 0.017 & 0.002 & 0.104 \\
\hline September & 0.41 & 0.14 & 0.18 & $<0.001$ & $<0.001$ & 0.006 \\
\hline October & 0.78 & 0.32 & 0.09 & $<0.001$ & $<0.001$ & $<0.001$ \\
\hline$P$ & $* *$ & $* * 3$ & $* *$ & & & \\
\hline
\end{tabular}

'Light (L)-stocked at 1.2 AUM/ha; Very Heavy (VH)-stocked at 4.8 AUM/ha.

${ }^{2}$ Level of significance $(*=P<0.05, * *=P<0.01$, NS $=P>0.05)$ of 2 nd degree polynomial regression of the constituent concentration over month.

${ }^{3}$ Ist degree polynomial regression.

Johnston et al. 1971, Willms et al. 1985). The vegetation changes, both qualitative and quantitative, on the grazed fields represent the effect by the various grazing pressures. Conversely, potential productivity of the vegetation within the exclosures will be affected by its protection (Tueller and Tower 1979). Naturally, the reduction in productivity of range plants resulting from a lack of grazing is always a legitimate concern when comparing grazed fields with exclosures. However, the difference particularly between field $L$ and its exclosure in terms of the results of the study under discussion will be quantitative rather than qualitative, since the only actual species composition differences (percent composition basal area) were trace vs. 0 and 0.2 vs trace for Artimisia frigida and
Oxytropis gracilis, respectively (Willms et al. 1985, Table 1 vs. Table 2).

Materials leached from aboveground live and dead herbage by rain-wash include a diversity of metabolically important compounds, such as amino acids, phenols, other organic acids and carbohydrates, and inorganic nutrients (Tukey 1969). Compounds synthesized by microbial activity will, of course, also be part of the leachates. The plant materials in this study, representing dead herbage only, were macerated. These water extracts of litter, therefore, represented the maximum potential effect of the chemical composition of precipitation percolating through dead herbage on rangeland soils. Although grazing superimposes other sets of 
Table 4. Regression coeffiejents in the concentration of chemical constituents between litter $(x)$ and soil $(y)$ after removing the effect of month', for the period from May to October.

\begin{tabular}{|c|c|c|c|c|c|c|}
\hline \multirow[b]{3}{*}{ Chemical constituent } & \multirow{2}{*}{\multicolumn{2}{|c|}{ Exclosure }} & \multicolumn{4}{|c|}{ Grazed } \\
\hline & & & \multicolumn{2}{|c|}{ Light } & \multicolumn{2}{|c|}{ Very heavy } \\
\hline & Coefficient & $\boldsymbol{P}$ & Coefficient & $P$ & Coefficient & $P$ \\
\hline Total C & -0.267 & 0.230 & -0.225 & 0.633 & 0.088 & 0.796 \\
\hline $\begin{array}{l}\text { Water-extractable C } \\
\text { (\% of total C) }\end{array}$ & -0.022 & 0.482 & 0.079 & 0.254 & 0.106 & 0.027 \\
\hline Total N (\%) & -0.235 & 0.304 & 0.059 & 0.784 & 0.498 & 0.211 \\
\hline $\begin{array}{l}\text { Water-extractable } N \\
\text { (\% of total } N)\end{array}$ & 0.026 & 0.199 & -0.077 & 0.040 & 0.063 & 0.131 \\
\hline $\begin{array}{l}\text { Organic acids } \\
\text { (at pH as extracted) }\end{array}$ & 0.484 & 0.001 & 0.047 & 0.804 & 0.405 & 0.010 \\
\hline $\begin{array}{l}\text { Organic acids } \\
\text { (at pH 2.5) }\end{array}$ & -0.715 & 0.001 & 0.252 & 0.335 & -0.048 & 0.785 \\
\hline $\begin{array}{l}\text { Palmitic acid } \\
\text { (at pH 2.5) }\end{array}$ & 0.621 & 0.079 & 0.577 & 0.224 & -0.278 & 0.552 \\
\hline $\begin{array}{l}\text { Stearic acid } \\
\text { (at pH 2.5) }\end{array}$ & 0.430 & 0.235 & -0.545 & 0.174 & 1.021 & 0.009 \\
\hline $\begin{array}{l}\text { Arachidic acid } \\
\text { (at pH 2.5) }\end{array}$ & 0.034 & 0.849 & 0.054 & 0.802 & 0.098 & 0.691 \\
\hline
\end{tabular}

The effect of month was significant $(P<0.01)$ in each case.

dynamics through additions of saliva and excreta, and through trampling, which exposes cell contents of fresh tissue, and through the change in species composition, none of these effects were considered in this study.

No water-extractable carbohydrates were present in the soils under consideration. Free sugars provide a rich source of available energy for metabolic use by microorganisms. Litter in July and August produced the greatest amount of water-extractable carbohydrates. Only traces of free sugars in water extracts were extractable from the Chernozemic soil studied, since the sugars are immediately incorporated into microbial tissue, mineralized, polymerized, or adsorbed on the soil itself. However, Gupta (1967) and Haynes and Swift (1990) did find small amounts of free sugars in water extracts of soils formed in higher rainfall areas.

Although many phenolic acids have been found in leachates from a number of grasses (Whitehead et al. 1982, Hartley and Whitehead 1985), Shindo and Kuwatsuka (1975) established that they were rapidly adsorbed by the soil. That is, once the throughfall has reached the soil, its contents are subjected to various processes that reduce or eliminate their concentration in the soil solution. To extract phenols and carbohydrates from the soil, more drastic extractions, such as the use of hot water at $80^{\circ} \mathrm{C}$ (Haynes and Swift 1990) or of $\mathrm{NaOH}$ or $\mathrm{H}_{2} \mathrm{SO}_{4}$, are required (Whitehead et al. 1981, Dormaar 1984).

Wang et al. (1971) found the predominant free fatty acids under various crops to be myristic, palmitic, palmitoleic, stearic, oleic and arachidic. Three major fatty acid peaks were present in the present study. Two were palmitic and stearic acid; the third peak was tentatively accepted as arachidic acid.

The soil close to the surface is a sink for nutrients (Christie 1979) and likely also for organic compounds leached into the soil from standing and fallen litter (Dormaar and Willms 1990). Repeated overgrazing reduces litter yield (Christie 1979, Willms 1988). Consequently, the quantity of $\mathrm{C}, \mathrm{N}$, organic acid, and carbohydrate mass entering the soil of field $\mathrm{VH}$ was considerably smaller than that in field $\mathbf{L}$ or in the exclosures.

Water-soluble constituents followed a nonlinear relationship over time with a maximum occurring in midsummer. Although site differences for specific months existed, the trends among sites were generally the same. There were also differences over the season for total leachable palmitic and arachidic acids, particularly between the exclosures and the grazed fields. That is, rain-induced leachates from plants differ between grazing pressures, and thus litter quality differs due to the various grazing pressures. Observations at regular time interval are therefore essential.

The relationship of constituents in soil vs. litter was less clear. Although water-extractable constituents from litter sometimes affected the equivalent soil chemical properties, either positively or negatively, no predictions on litter or soil constituents were possible under the conditions of the experiment.

AlSaadawi et al. (1983) showed that among the long-chain fatty acids extracted from Polygonum aviculare residue, palmitic, stearic, and arachidic acids had strong allelopathic action against various test plants. The organic acids obtained at pH 2.5 were released from larger complexes by acid hydrolysis. In reality, they would thus be somewhat protected and released, if at all, more slowly into the soil solution. Nevertheless, the significantly greater quantities of water-extractable organic acids, at $\mathrm{pH} 2.5$, taken from soil in grasslands that are in good condition because of low grazing pressure (Table 2: E vs. L and E vs. VH) might well contribute to the resistance of those grasslands to encroachment by invading species. For a deeper understanding as to the ecological balance between litter leachate and the organization of natural and transformed plant communities both root exudates and litter leachates of individual plants will have to be evaluated.

\section{Literature Cited}

AlSandawi, I.S., E.L. Rice, and T.K.B. Karns. 1983. Allelopathic effects of Polygonum aviculare L. III. Isolation, characterization, and biological activities of phytotoxins other than phenols. J. Chem. Ecol. 9:761-774.

Association of Official Agricultural Chemists. 1950. Official methods of analysis. 7th ed., Washington, D.C.

Bokhari, U.G. 1978. Allelopathy among prairie grasses and its possible ecological significance. Ann. Bot. 42:127-136.

Christie, E.K. 1979. Ecosystem processes in semiarid grasslands. II. Litter production, decomposition and nutrient dynamics. Austr. J. Agr. Res. 30:29-42.

Coupland, R.T., and F.C. Brayshaw. 1953. The fescue grassland of Saskatchewan. Ecology 34:386-405.

Dormaar, J.F. 1984. Monosaccharides in hydrolysates of water-stable aggregates after 67 years of cropping to spring wheat as determined by capillary gas chromatography. Can. J. Soil Sci. 64:647-656.

Dormaar, J.F., and U.J. Pittman. 1980. Decomposition of organic residues as affected by various dryland spring wheat-fallow rotations. Can. J. Soil Sci. 60:97-106.

Dormaar, J.F., and W.D. Wilms. 1990. Effect of grazing and cultivation on chemical properties of soils in the mixed prairie. J. Range Manage. 43:456-460. 
Dormaar, J.F., A. Johnston, and S. Smoliak. 1984. Seasonal changes in carbon content, and dehydrogenase, phosphatase, and urease activities in mixed prairie and fescue grassland Ah horizons. J. Range Manage. 37:31-35.

Gilliam, F.S. 1987. The chemistry of wet deposition for a tallgrass prairie ecosystem: Inputs and interactions with plant canopies. Biogeochemistry 4:203-217.

Gupta, U.C. 1967. Carbohydrates, p. 91-118. In: A.D. McLaren and G.H. Peterson (eds.), Soil Biochemistry. Marcel Dekker, Inc., New York.

Haynes, R.J., and R.S. Swift. 1990. Stability of soil aggregates in relation to organic constituents and soil water content. J. Soil Sci. 41:73-83.

Hartley, R.D., and D.C. Whitehead. 1985. Phenolic acids in soils and their influence on plant growth and soil microbial processes, p. 109-149. In: D. Vaughan and R.E. Malcolm (eds.), Soil Organic Matter and Biological Activity. Martinus Nijhoff/Dr. W. Junk Publishers, Dordrecht, Netherlands.

Johnston, A. 1961. Some factors affecting germination, emergence, and early growth of three range grasses. Can. J. Plant Sci. 41:59-70.

Johnston, A., J.F. Dormaar, and S. Smoliak. 1971. Long-term grazing effects on fescue grassland soils. J. Range Manage. 24:185-188.

Knapp, A.K., and T.R. Seastedt. 1986. Detritus accumulation limits productivity of tall grass prairie. BioScience 36:662-668.

Peake, R.W., and A. Johnston. 1965. Grazing effects on fescue grassland in western Canada. Proc. 9th Int. Grassl. Congr., Brazil: 1627-1630.

Rice, E.L. 1984. Allelopathy. 2nd ed. Academic Press, Inc., N.Y.

Ridley, A.D., and R.A. Hedlin. 1968. Soil organic matter and crop yields as influenced by the frequency of summerfallowing. Can. J. Soil Sci. 48:315-322.
Shindo, H., and S. Kuwatsuka. 1975. Behavior of phenolic substances in the decaying process of plants. III. Degradation pathway of phenolic acids. Soil Sci. Plant Nutr. 21:227-238.

Steel, R.G.D., and J.H. Torrie. 1980. Principles and procedures of statistics. A biometrical approach. 2nd ed. McGraw-Hill, Toronto.

Tueller, P.T., and J.D. Tower. 1979. Vegetation stagnation in three-phase big game exclosure. J. Range Manage. 32:128-203.

Tukey, Jr., H.B. 1969. Implications of allelopathy in agricultural plant science. Bot. Rev. 35:1-16.

Wang, T.S.C., P.T. Hwang, and C.Y. Chen. 1971. Soil lipids under various crops. Soil Sci. Soc. Amer. Proc. 35:584-587.

Whitehead, D.C., H. Dibb, and R.D. Hartley. 1981. Extractant pH and the release of phenolic compounds from soils, plant roots and leaf litter. Soil Biol. Biochem. 13:343-348.

Whitehead, D.C., H. Dibb, and R.D. Hartley. 1982. Phenolic compounds in soil as influenced by the growth of different plant species. J. Appl. Ecol. 19:579-588.

Whittaker, R.H. 1970. The biochemical ecology of higher plants p. 43-70. In: E. Sandheimer and J.B. Simeone (eds.), Chemical Ecology. Academic Press, N.Y.

Willms, W.D. 1988. Response of rough fescue (Festuca scabrella) to light, water, temperature, and litter removal, under controlled conditions. Can. J. Bot. 66:429-434.

Willms, W.D., S. Smoliak, and A.W. Bailey. 1986. Herbage production following litter removal on Alberta native grasslands. J. Range Manage. 39:536-540.

Willms, W.D., S. Smoliak, and J.F. Dormaar. 1985. Effects of stocking rate on a rough fescue grassland vegetation. J. Range Manage. 38:220-225. 